\title{
Association between Physical Activity and Health-Related Quality of Life in Korean: The Korea National Health and Nutrition Examination Survey IV

\section{Sunmi Kim, Young-Min Kwon ${ }^{1, *}$, You-Il Park ${ }^{1}$}

Department of Family Medicine, Kangwon National University Hospital, Chuncheon; ${ }^{1}$ Department of Family Medicine, Sahmyook Medical Center, Seoul, Korea

Background: Numerous studies have identified that physical activity (PA) has positive influences on quality of life. However, there has been little domestic research about the relation of PA to health-related quality of life (HRQoL) among the general population. The purpose of this study was to evaluate the effect of PA on HRQoL in Korean adults aged 40 years or over.

Methods: This cross-sectional study was based on data acquired from the Korean National Health and Nutrition Examination Survey IV. Final study subjects were 10,392 adults aged 40 years or over. According to the severity and frequency of PA, the subjects were divided into three PA groups as follows: high-activity, mid-activity, and low-activity group. We compared the HRQoL estimated by the EuroQoL 5-dimension (EQ-5D), the EQ visual analogue scale (EQ-VAS), and EQ-5D index between three PA groups.

Results: The odds ratios for mid-activity group and high activity group compared with low activity group were significantly decreased in the dimension of mobility of EQ-5D (mid-activity group, 0.81 [0.69-0.94]; high activity group, 0.81 [0.67-0.99]). EQ-VAS was decreased as PA level was lowered from high activity group and mid-activity to low activity group. EQ-5D index was the highest in mid-activity group and the lowest in low activity group.

Conclusion: PA above mid-activity group showed better HRQoL compared than low activity group.

Keywords: Motor Activity; Quality of Life; Population; Health

Received: August 30, 2011, Accepted: April 28, 2014

${ }^{*}$ Corresponding Author: Young-Min Kwon

Tel: +82-2-2210-3245, Fax: +82-2-2212-2673

E-mail: Kym0177@hanmail.net

Korean Journal of Family Medicine

Copyright (C) 2014 The Korean Academy of Family Medicine

(a) This is an open-access article distributed under the terms of the Creative Commons Attribution Non-Commercial License (http://creativecommons.org/licenses/by-nc/3.0) which permits unrestricted noncommercial use, distribution, and reproduction in any medium, provided the original work is properly cited.

\section{INTRODUCTION}

Regular physical activity (PA) can reduce the risk of many adverse health outcomes. ${ }^{1)}$ It has been reported that PA can lower risk of early death, coronary heart disease, stroke, high blood pressure, type 2 diabetes, and dyslipidemia. ${ }^{1)}$ A longitudinal study showed that every additional 15 minutes of daily PA up to 100 minutes per day resulted in a further $4 \%$ decrease in mortality from any cause. ${ }^{2)}$ Moreover, PA can also reduce depression and produce better cognitive function. ${ }^{1)}$ PA can play a role in 
maintaining functional ability later in life, reduce disability and improve quality of life. ${ }^{3,4)}$ Since 1948, when the World Health Organization defined health as being not only the absence of disease and infirmity but also the presence of physical, mental, and social well-being, ${ }^{5)}$ quality of life issues have become steadily more important in health care practice. ${ }^{6)}$ Impaired health-related quality of life (HRQoL) has been associated with increased mortality and disease progression. ${ }^{7,8)}$

Previous studies have reported that PA has been positively linked to quality of life, ${ }^{9,10)}$ however, there is insufficient domestic data on the association between PA and HRQoL among the general population. Therefore, in this study, we evaluated whether PA is associated with HRQoL in the Korean adult population who aged 40 years or over, using nationally representative Korean population data from the Korean National Health and Nutrition Examination Survey (KNHANES) IV.

\section{METHODS}

\section{Study Subjects}

This cross-sectional study was based on data acquired from the KNHANES IV conducted by the Korea Center for Disease Control and Prevention in 2007, 2008, and 2009. The survey included a Health Interview Survey, a Health Behavior Survey, a Health Examination Survey, and a Nutrition Survey. KNHANES IV adopted the stratified multistage cluster sampling design by using the rolling-survey sampling method. ${ }^{11)}$ Therefore, the rolling sample collected each year is the probability sample representing the general Korean population. The institutional review board at the Korea Centers for Disease Control and Prevention approved the protocol, and all participants signed informed consent forms. Of the 23,317 participants of the Health Interview Survey, a Health Behavior Survey, and a Health Examination Survey, we included 11,393 subjects aged 40 years or older. Among the 11,393 subjects, 664 subjects who did not respond to the questionnaire on HRQoL were excluded. Another 337 subjects with incomplete data on social economic status such as income and education level were also excluded. Thus, a final total of 10,392 participants were eligible for our study.

\section{Assessment of Physical Activity}

PA was assessed using the Korean version of the International Physical Activity Questionnaire acquired by the Health Interview Survey. ${ }^{12)}$ Participants were asked questions regarding their PA level during a normal week. In terms of vigorous PA, participants were asked: "during the last 7 days, on how many days did you do vigorous PA (for at least 10 minutes)? (vigorous PA: running, mountain climbing, soccer, basketball, or any other activity that causes a substantial increase in breathing or heart rate).” Concerning moderate PA, participants were asked the following: "during the last 7 days, on how many days did you do moderate PA (for at least 10 minutes)? (moderate PA: tennis doubles, volleyball, badminton or table tennis, or any other activity that causes a slight increase in breathing or heart rate).” For each type of activity, the participants indicated the average total time per day devoted to the activity. Level of PA was classified into three groups: high activity group, mid-activity group, and low activity group. Subjects who performed vigorous PA for at least 20 minutes per day on 3 days per week were assigned to the high activity group. Subjects who performed moderate PA for at least 30 minutes per day on 5 days per week were assigned to the midactivity group. The remaining subjects were assigned to the low activity group.

\section{Assessment of Health-Related Quality of Life}

HRQoL was evaluated using the EuroQol, which consists of two parts, the health-status descriptive system (EuroQoL 5-dimension, EQ-5D) and the EQ visual analogue scale (EQVAS). The EQ-5D records the level of self-reported problems according to five dimensions (mobility, self-care, usual activities, pain/discomfort, and anxiety/depression). ${ }^{13,14)}$ Each of the dimensions is assessed based on a single question with three response levels (no problem, some problems, and extreme problems). Using a combination of these items, a single health index was calculated using the Korea valuation set developed by the Korean Centers for Disease Control and Prevention. ${ }^{15)}$ Scores on the EQ-5D index range from -0.171 to 1 , where 1 indicates no problem in any of the five dimensions, zero indicates death, and negative values indicate a health status worse than death. Respondents described their own health status using a VAS ranging from 0 (worst imaginable health) to 100 (best imaginable health), which is represented as EQ-VAS. ${ }^{14)}$ The Korean version 
of the EQ-5D had already been made and evaluated for its validity and reliability. ${ }^{16)}$

\section{Other Potential Confounding Variables}

Trained medical staff measured height and weight by 0.1 $\mathrm{cm}$ and $0.1 \mathrm{~kg}$, respectively, following standardized procedures at mobile examination centers. Body mass index (BMI, kg/ $\mathrm{m}^{2}$ ) was calculated as weight divided by height squared ( $\mathrm{kg} /$ $\mathrm{m}^{2}$ ). Socio-demographic data, including sex, age, household income, education level, marriage status, smoking status, alcohol consumption, and restricted activity was obtained using selfadministered questionnaires. Participants were also asked whether they had ever been told by a physician that they had hypertension, diabetes mellitus, stroke, heart diseases (acute myocardial infarction or angina), arthritis (osteoarthritis or rheumatoid arthritis), or any malignancy. Household income were divided into four groups (lowest, lower intermediate, higher intermediate, and highest groups) as was education level (elementary school, middle school, high school, and higher than university). Marriage status was reported as either single or married. On the basis of smoking status, participants were classified as never smokers, former smokers (had smoked $\times 100$ cigarettes during their lifetime but were not smoking currently) and current smokers (had smoked $\times 100$ cigarettes and were still smoking). Participants who drink alcohol more than once a week were regarded as problem drinkers. Hemoglobin concentrations were measured by ADVIA 120 (Siemens Healthcare Diagnostics, Tarrytown, NY, USA) and XE-2100D (Sysmex, Kobe, Japan). Anemia was diagnosed when hemoglobin was less than $13 \mathrm{~g} / \mathrm{dL}$ for men and less than $12 \mathrm{~g} / \mathrm{dL}$ for women.

\section{Statistical Analysis}

All statistical analyses were conducted with Stata SE ver. 10.0 (Stata Co., College Station, TX, USA) using the survey data commands (svy) to account for cluster effects and sampling weights. Means and standard deviations (mean \pm SDs) or the number and proportions ( $\mathrm{n}[\%]$ ) were presented. The baseline characteristics, the categories of EQ-5D and EQ-VAS of the participants were compared according to PA group using oneway analysis of variance for continuous variables and Pearson' s chi-square test with Rao-Scott correction using F statistic for categorical variables. After adjusting the confounding variables including age, sex, household income, education level, marriage status, problem drinker, smoking status, BMI, past medical history, and restricted activity, we conducted multiple logistic regression analyses to estimate odds ratios (ORs) of the discomfort in each five categories of EQ-5D (mobility, selfcare, usual activities, pain/discomfort, and anxiety/depression) according to PA level. After adjusting the confounding variables including age, sex, household income, education level, marriage status, drinking, smoking status, BMI, past medical history, and restricted activity, we also estimated and compared the adjusted means and 95\% confidence intervals of both EQ-VAS and EQ5D index in each low, mid- and high activity group by using analysis of covariance. All statistical significance was defined by a two-tailed P-value $<0.05$.

\section{RESULTS}

\section{Baseline Characteristics According to Physical Activity Groups}

Baseline characteristics of the participants classified by PA are shown in Table 1 . The mid-activity group had the highest mean age $(59.7 \pm 11.8)$, and high activity group had the lowest mean age (56.2 \pm 10.8$)$. The PA group with higher activity comprised a higher proportion of men $(45.7 \%, 43.6 \%$, and $40.1 \%$, respectively). The high activity group had a higher proportion of subjects with highest household income and with spouse. The high activity group had more problem drinkers and higher BMI $\left(\mathrm{kg} / \mathrm{m}^{2}\right)$, however, the proportion of current smokers was not significantly different between three PA groups. Prevalence of hypertension, stroke, and arthritis was the lowest in high activity group. Prevalence of malignancy, diabetes, cardiovascular disease, and anemia was not different between three PA groups. The proportion of the subjects with restricted activity was the highest in low activity group (Table 1 ).

\section{Distribution of EuroQoL 5-Dimension and EQ Visual Analogue Scale According to Physical Activity Groups \\ HRQoL evaluated by EQ-5D and EQ-VAS according to PA} groups was shown in Table 2 . The high activity group had the highest percentage of subjects who responded "no problem" in 
Table 1. Baseline characteristics according to physical activity groups

\begin{tabular}{|c|c|c|c|c|}
\hline Physical activity & Low activity group $(\mathrm{n}=4,494)$ & Mid-activity group $(\mathrm{n}=3,219)$ & High activity group $(\mathrm{n}=2,679)$ & P-value* \\
\hline Age (y) & $58.3 \pm 12.5$ & $59.7 \pm 11.8$ & $56.2 \pm 10.8$ & $<0.01$ \\
\hline Sex (men) & $1,802(40.1)$ & $1,402(43.6)$ & $1,224(45.7)$ & $<0.01$ \\
\hline Household income & & & & $<0.01$ \\
\hline Group I (lowest) & $1,314(29.2)$ & $1,001(31.1)$ & $573(21.4)$ & \\
\hline Group II & $1,100(24.5)$ & $817(25.4)$ & $688(25.7)$ & \\
\hline Group III & $992(22.1)$ & 705 (21.9) & $718(26.8)$ & \\
\hline Group IV (highest) & $1,088(24.2)$ & $696(21.6)$ & $700(26.1)$ & \\
\hline Education level & & & & $<0.01$ \\
\hline Elementary & $1,810(40.3)$ & $1,426(44.4)$ & $948(35.4)$ & \\
\hline Middle school & $732(16.3)$ & $496(15.4)$ & 495 (18.5) & \\
\hline High school & $1,131(25.2)$ & $798(24.8)$ & 765 (28.6) & \\
\hline Higher than university & 819 (18.2) & $495(15.4)$ & $470(17.6)$ & \\
\hline Marriage status (with spouse) & $3,411(75.9)$ & $2,480(77.0)$ & $2,228(83.2)$ & $<0.01$ \\
\hline Problem drinker & $722(16.1)$ & $516(16.0)$ & $553(20.6)$ & $<0.01$ \\
\hline Smoking status & & & & $<0.01$ \\
\hline Current smoker & $870(19.4)$ & $615(19.1)$ & $517(19.3)$ & \\
\hline Former-smoker & $890(19.8)$ & $727(22.6)$ & $625(23.3)$ & \\
\hline Non- smoker & $2,730(60.8)$ & $1,877(58.3)$ & $1,537(57.4)$ & \\
\hline Body mass index $\left(\mathrm{kg} / \mathrm{m}^{2}\right)$ & $24.0 \pm 3.2$ & $23.8 \pm 3.0$ & $24.2 \pm 3.1$ & 0.03 \\
\hline \multicolumn{5}{|l|}{ Past medical history } \\
\hline Hypertension & $1,351(30.1)$ & $980(30.4)$ & $706(26.4)$ & $<0.01$ \\
\hline Diabetes & $491(10.9)$ & $389(12.1)$ & $273(10.2)$ & 0.06 \\
\hline Stroke & $160(3.6)$ & $129(4.0)$ & $71(2.7)$ & 0.02 \\
\hline Cardiovascular disease & 149 (3.3) & $111(3.5)$ & 77 (2.9) & 0.43 \\
\hline Arthritis & $1,207(26.9)$ & $863(26.8)$ & $654(24.4)$ & 0.05 \\
\hline Malignancy & $170(3.8)$ & $147(4.6)$ & $103(3.8)$ & 0.19 \\
\hline Anemia & $496(11.0)$ & $342(10.6)$ & $249(9.3)$ & 0.06 \\
\hline Restricted activity & $1,285(28.6)$ & $829(25.8)$ & $933(19.9)$ & $<0.01$ \\
\hline
\end{tabular}

Values are presented as mean \pm SD or number (\%).

*By one-way analysis of variance or chi-square analyses.

all five dimensions of EQ-5D (mobility, self-care, usual activities, pain/discomfort, and anxiety/depression), and the lowest percentage of subjects who responded "extreme problems" in four dimensions of EQ-5D (mobility, self-care, usual activities, and pain/discomfort). The mid-activity group had the lowest proportion of subjects who responded "extreme problems" in the dimension of anxiety/depression of EQ-5D. The mean of
EQ-VAS was the highest in high activity group and the lowest in low activity group (high activity group, $75.3 \pm 16.8$; mid-activity group, $72.7 \pm 18.8$; low activity group, $69.5 \pm 20.6$ ) (Table 2 ).

\section{Comparison of Health-Related Quality of Life between Physical Activity Groups}

By using multiple logistic regression analyses after adjusting 
Table 2. Distribution of EQ-5D and EQ-VAS according to physical activity groups

\begin{tabular}{|c|c|c|c|c|}
\hline Physical activity & Low activity group $(\mathrm{n}=4,494)$ & Mid-activity group $(n=3,219)$ & High activity group $(n=2,679)$ & P-value \\
\hline \multicolumn{5}{|l|}{ EQ-5D level* } \\
\hline \multicolumn{5}{|l|}{ Mobility } \\
\hline 1 & $3,157(70.3)$ & $2,382(74.0)$ & $2,125(79.3)$ & $<0.01^{\dagger}$ \\
\hline 2 & $1,258(28.0)$ & $824(25.6)$ & $547(20.4)$ & \\
\hline 3 & $79(1.8)$ & $13(0.4)$ & $7(0.3)$ & \\
\hline \multicolumn{5}{|l|}{ Self-care } \\
\hline 1 & $4,098(91.2)$ & $2,996(93.1)$ & $2,563(95.7)$ & $<0.01^{\dagger}$ \\
\hline 2 & $352(7.8)$ & $205(6.4)$ & $111(4.1)$ & \\
\hline 3 & $44(1.0)$ & $18(0.6)$ & $5(0.2)$ & \\
\hline \multicolumn{5}{|l|}{ Usual activities } \\
\hline 1 & $3,592(79.9)$ & $2,665(82.8)$ & $2,321(86.6)$ & $<0.01^{\dagger}$ \\
\hline 2 & $776(17.3)$ & $495(15.4)$ & $329(12.3)$ & \\
\hline 3 & $126(2.8)$ & $59(1.8)$ & $29(1.1)$ & \\
\hline \multicolumn{5}{|l|}{ Pain/discomfort } \\
\hline 1 & $2,826(62.9)$ & $2,116(65.7)$ & $1,838(68.6)$ & $<0.01^{\dagger}$ \\
\hline 2 & $1,369(30.5)$ & $937(29.1)$ & $723(27.0)$ & \\
\hline 3 & 299 (6.7) & $166(5.2)$ & $118(4.4)$ & \\
\hline \multicolumn{5}{|c|}{ Anxiety/depression } \\
\hline 1 & $3,697(82.3)$ & 2,701 (83.9) & 2,291 (85.5) & $<0.01^{\dagger}$ \\
\hline 2 & $712(15.8)$ & $472(14.7)$ & $349(13.0)$ & \\
\hline 3 & $85(1.9)$ & $46(1.4)$ & $39(1.5)$ & \\
\hline EQ-VAS & $69.5 \pm 20.6$ & $72.7 \pm 18.8$ & $75.3 \pm 16.8$ & $<0.01^{\ddagger}$ \\
\hline
\end{tabular}

Values are presented as mean \pm SD or number (\%).

EQ-5D: EuroQoL 5-dimension, EQ-VAS: EQ visual analogue scale.

*Level of EQ-5D was as follows: 1: no problem, 2: some problems, 3: extreme problems. ${ }^{\dagger}$ By chi-square analyses. ${ }^{\ddagger}$ By one-way analysis of variance.

for confounding variables including age, sex, household income, education level, marriage status, problem drinking, smoking status, BMI, past medical history, and restriction of activity, the ORs for mid-activity group and high activity group compared with low activity group were significantly decreased in the dimension of mobility of EQ-5D (mid-activity group, 0.81 [0.69-0.94]; high activity group, 0.81 [0.67-0.99]). The analysis of covariance (ANCOVA) analysis after controlling the covariates including age, sex, household income, education level, marriage status, problem drinking, smoking status, BMI, past medical history, and restriction of activity showed that the adjusted mean of EQ-VAS was decreased as PA level was lowered from high activity group and mid-activity to low activity group (high activity group, 74.1 [73.4-74.8]; mid-activity group, 73.1 [72.5-73.7]; low activity group, 70.0 [69.4-70.5]; P-value < 0.05). By ANCOVA analysis as above, the adjusted mean of EQ-5D index was the highest in mid-activity group and the lowest in low activity group, and the difference between adjusted means of three PA groups was statistically significant (Table 3).

\section{DISCUSSION}

In this study, we examined the relationship between PA 
Table 3. Comparison of health-related quality of life between physical activity groups by using multiple logistic regression analyses and analysis of covariance

\begin{tabular}{|c|c|c|c|c|c|}
\hline \multirow{2}{*}{ Variable } & \multirow{2}{*}{$\begin{array}{l}\text { Low activity group } \\
\qquad(\mathrm{n}=4,494)\end{array}$} & \multicolumn{2}{|c|}{ Mid-activity group $(\mathrm{n}=3,219)$} & \multicolumn{2}{|c|}{ High activity group $(\mathrm{n}=2,679)$} \\
\hline & & $\mathrm{OR}^{*}(95 \% \mathrm{CI})$ & P-value ${ }^{\dagger}$ & $\mathrm{OR}^{*}(95 \% \mathrm{CI})$ & P-value ${ }^{\dagger}$ \\
\hline \multicolumn{6}{|l|}{ EQ-5D } \\
\hline Mobility & 1.00 (reference) & $0.81(0.69-0.94)$ & 0.007 & $0.81(0.67-0.99)$ & 0.036 \\
\hline Self-care & 1.00 (reference) & $0.79(0.57-1.02)$ & 0.073 & $0.77(0.57-1.04)$ & 0.086 \\
\hline Usual activities & 1.00 (reference) & $0.80(0.64-0.97)$ & 0.025 & $0.93(0.73-1.20)$ & 0.58 \\
\hline Pain/discomfort & 1.00 (reference) & $0.93(0.80-1.08)$ & 0.343 & $0.99(0.83-1.18)$ & 0.892 \\
\hline Anxiety/depression & 1.00 (reference) & $1.03(0.86-1.23)$ & 0.728 & $0.92(0.78-1.08)$ & 0.305 \\
\hline EQ-VAS & $70.0(69.4-70.5)$ & $73.1(72.5-73.7)$ & & $74.1(73.4-74.8)$ & \\
\hline EQ-5D index & $0.868(0.863-0.872)$ & $0.885(0.880-0.890)$ & & $0.880(0.874-0.885)$ & \\
\hline
\end{tabular}

OR: odds ratio, CI: confidence interval, EQ-5D: EuroQoL 5-dimension, EQ-VAS: EQ visual analogue scale.

${ }^{*}$ ORs for any discomfort. ${ }^{\dagger}$ P-value $<0.05$; after adjusting for the confounding variables including age, sex, household income, education level, marriage status, problem drinking, smoking status, body mass index, past medical history, and restriction of activity.

level and HRQoL by using a nationally representative sample of Korean adults who were 40 years or older. The adjusted means of EQ-VAS and EQ-5D index after controlling the covariates were the lowest in the low activity group compared with mid-activity and high activity group, showing the positive association between PA level and HRQoL. The distribution of EQ-5D according to PA level, the high activity group had the highest proportion of 'no problem' and the lowest proportion of 'extreme problem' in all dimensions of EQ-5D (mobility, self-care, usual activity, pain/ discomfort, and anxiety/depression). However, after adjusting the covariates, the association of PA level with the dimensions of usual activity, pain/discomfort, and anxiety/depression of EQ-5D was not statistically significant. High activity group had significantly higher adjusted mean compared with low activity group only in the mobility dimension of EQ-5D and mid-activity group had significantly higher adjusted means compared with low activity group in the dimensions of mobility and usual activities of EQ-5D, which might affect the associations of PA level with EQVAS and EQ-5D Index. It is suggested that the conditions that encourage regular PA possible had a positive effect on HRQoL, which is partly consistent with previous studies. ${ }^{17-20)}$ A previous study reported that recommended levels of PA were related with elevated HRQoL; however, participation in daily moderate or vigorous $\mathrm{PA}$ and participation in very short or extended periods of PA was associated with poorer HRQOL. ${ }^{17)}$ Other studies showed that the participants who regularly participated in PA of at least moderate intensity had higher HRQoL than those who were less physically active. ${ }^{18,19)}$ In this study, the dimension of mobility and usual activities might mainly contribute to the significant association of PA with EQ-VAS and EQ-5D index, suggesting that regular PA itself was not directly related with symptom of anxiety or depression and could have an indirect effect via the circumstance that make regular PA possible. However, several epidemiological studies have documented an association between PA and anxiety/depression. ${ }^{21,22)}$ Although the association of $\mathrm{PA}$ and the prevalence of depression and anxiety disorders has been repeatedly described, few studies examined the association of PA with mental problems prospectively. ${ }^{23)}$ In addition, the relationship between PA and the dimension of pain/discomfort was not significant in our study result, which was inconsistent with the previous study that reported PA level can influence pain, pain interference and fatigue in 116 elders with chronic pain. ${ }^{24)}$ The inconsistency could result from the difference in study subjects, design and methods of PA measurement.

Due to the overall design of this study, several limitations exist. First, this is a cross-sectional study, which limited its ability to establish a causal relationship between PA and HRQoL. Further prospective studies are needed to verify the causal relationship between PA and HRQoL. Second, there was potential recall bias because PA and other lifestyle factors were based on information 
retrospectively collected by self-reported questionnaires. Third, EQ-5D had limitation for the measurement of HRQoL. Some reports have suggested in that a 36-item short-form is more sensitive than the EQ-5D in healthy people and for detecting small health changes. ${ }^{25,26)}$ However, algorithms based on survey tools including more comprehensive aspects of HRQoL did not seem to measure greater variations in utility than those based on simpler tools such as the EQ-5D. Moreover, the EQ-5D is easier to use and less time-consuming. ${ }^{27)}$ Hence the EQ-5D has some advantages for large population studies such as the KNHANES.

In summary, PA is important for the improvement of health status as well HRQoL. Not only emphasizing the effect of regular PA, but attempting to understand and correct the circumstances which may disturb regular PA can help to improve HRQoL.

\section{CONFLICT OF INTEREST}

No potential conflict of interest related with this article was reported.

\section{ACKNOWLEDGMENTS}

This study was funded by Clinical Research Center of Kangwon National University Hospital.

\section{REFERENCES}

1. U.S. Department of Health and Human Services. Physical activity guidelines for Americans [Internet]. Washington (DC): U.S. Department of Health and Human Services; 2008 [cited 2014 April 1]. Available from: http://www.health.gov/ paguidelines/.

2. Wen CP, Wai JP, Tsai MK, Yang YC, Cheng TY, Lee MC, et al. Minimum amount of physical activity for reduced mortality and extended life expectancy: a prospective cohort study. Lancet 2011;378:1244-53.

3. Brach JS, FitzGerald S, Newman AB, Kelsey S, Kuller L, VanSwearingen JM, et al. Physical activity and functional status in community-dwelling older women: a 14-year prospective study. Arch Intern Med 2003;163:2565-71.

4. Division of Ageing and Seniors. Physical activity and older adults from Canada [Internet]. Winnipeg: Public Health Agency of Canada; 2011 [cited 2014 Apr 1]. Available from: http://www.phac-aspc.gc.ca/seniors-aines/index-eng.php.

5. World Health Organization. Constitution of the World Health Organization. In: World Health Organization, editor. Handbook of basic documents. 5th ed. Geneva: Palais des Nations; 1952.p. 3-20.

6. Testa MA, Simonson DC. Assesment of quality-of-life outcomes. N Engl J Med 1996;334:835-40.

7. Kleefstra N, Landman GW, Houweling ST, Ubink-Veltmaat LJ, Logtenberg SJ, Meyboom-de Jong B, et al. Prediction of mortality in type 2 diabetes from health-related quality of life (ZODIAC-4). Diabetes Care 2008;31:932-3.

8. Schenkeveld L, Pedersen SS, van Nierop JW, Lenzen MJ, de Jaegere PP, Serruys PW, et al. Health-related quality of life and long-term mortality in patients treated with percutaneous coronary intervention. Am Heart J 2010;159:471-6.

9. Choi H, Lee EO, Kim BW, Kim IJ. Stage of change for exercise and health-related quality of life in Korean adults. J Korean Acad Adult Nurs 2004;16:191-201.

10. Lee GH, Kim CH, Shin HC, Park YW, Sung EJ. The relation of physical activity to health related quality of life. J Korean Acad Fam Med 2007;28:451-9.

11. Korea Centers for Disease Control and Prevention. The fourth national health and nutrition examination survey (KHANES IV). Cheongwon: Korea Centers for Disease Control and Prevention; 2010.

12. Craig CL, Marshall AL, Sjostrom M, Bauman AE, Booth $\mathrm{ML}$, Ainsworth BE, et al. International physical activity questionnaire: 12 -country reliability and validity. Med Sci Sports Exerc 2003;35:1381-95.

13. EuroQol Group. EuroQol: a new facility for the measurement of health-related quality of life. Health Policy 1990;16:199208.

14. Brooks R. EuroQol: the current state of play. Health Policy 1996;37:53-72.

15. Nam HS, Kim KY, Kwon SS, Koh KW, Poul K. EQ5D Korean valuation study using time trade of method. Cheongwon: Korea Centers for Disease Control and Prevention; 2007. 
16. Kim MH, Cho YS, Uhm WS, Kim S, Bae SC. Cross-cultural adaptation and validation of the Korean version of the EQ5D in patients with rheumatic diseases. Qual Life Res 2005; 14:1401-6.

17. Brown DW, Brown DR, Heath GW, Balluz L, Giles WH, Ford ES, et al. Associations between physical activity dose and health-related quality of life. Med Sci Sports Exerc 2004; 36:890-6.

18. Acree LS, Longfors J, Fjeldstad AS, Fjeldstad C, Schank B, Nickel KJ, et al. Physical activity is related to quality of life in older adults. Health Qual Life Outcomes 2006;4:37.

19. Vuillemin A, Boini S, Bertrais S, Tessier S, Oppert JM, Hercberg S, et al. Leisure time physical activity and healthrelated quality of life. Prev Med 2005;41:562-9.

20. Morimoto T, Oguma Y, Yamazaki S, Sokejima S, Nakayama T, Fukuhara S. Gender differences in effects of physical activity on quality of life and resource utilization. Qual Life Res 2006; 15:537-46.

21. De Moor MH, Boomsma DI, Stubbe JH, Willemsen G, de Geus EJ. Testing causality in the association between regular exercise and symptoms of anxiety and depression. Arch Gen
Psychiatry 2008;65:897-905.

22. Martinsen EW. Physical activity in the prevention and treatment of anxiety and depression. Nord J Psychiatry 2008; 62 Suppl 47:25-9.

23. Strohle A. Physical activity, exercise, depression and anxiety disorders. J Neural Transm 2009;116:777-84.

24. Cha BK, Park CS. A comparison of pain, pain interference and fatigue according to the level of physical activity in the elderly with chronic pain. J Korean Acad Community Health Nurs 2011;22:162-72.

25. Kopec JA, Willison KD. A comparative review of four preference-weighted measures of health-related quality of life. J Clin Epidemiol 2003;56:317-25.

26. Longworth L, Bryan S. An empirical comparison of EQ-5D and SF-6D in liver transplant patients. Health Econ 2003;12: 1061-7.

27. Glasziou P, Alexander J, Beller E, Clarke P; ADVANCE Collaborative Group. Which health-related quality of life score?: a comparison of alternative utility measures in patients with Type 2 diabetes in the ADVANCE trial. Health Qual Life Outcomes 2007;5:21. 